\title{
Adhesion G Protein-Coupled Receptor A2
}

National Cancer Institute

\section{Source}

National Cancer Institute. Adhesion G Protein-Coupled Receptor A2. NCI Thesaurus.

Code C150241.

Adhesion G protein-coupled receptor A2 (1338 aa, 143 kDa) is encoded by the human

ADGRA2 gene. This protein plays a role in central nervous system development and endothelial cell migration. 